\title{
Foramen oval restrictivo intraútero: causa de hipertensión pulmonar neonatal
}

\author{
Intrauterine restrictive foramen ovale: cause of neonatal pulmonary \\ hypertension
}

\author{
Lic. en Medicina Sandra Terroba Seara ${ }^{a}$, Lic. en Medicina Ignacio Oulego Erroz ${ }^{a}$, \\ Dr. en Medicina Carlos Lobete Prieto ${ }^{a}$ y Dra. en Medicina Paula Alonso Quintela ${ }^{a}$
}

\section{RESUMEN}

El cierre prematuro del foramen oval o foramen oval restrictivo intraútero es una entidad rara de etiología desconocida. Dicha patología se asocia a un riesgo elevado de hipertensión pulmonar y puede ocasionar insuficiencia cardíaca congestiva con hidrops y muerte fetal. También, se puede asociar con disfunción diastólica en el período neonatal.

Se presenta a un recién nacido en el que, en una ecografía prenatal, se visualizó un aumento de las cavidades derechas. En el ecocardiograma posnatal, se confirmó la dilatación de las cavidades derechas con hipertensión pulmonar en rango sistémico y septo interauricular inmóvil con foramen oval cerrado. A los 9 días, ante la persistencia de dificultad respiratoria, se realizó un ecocardiograma, con mejoría de la hipertensión pulmonar, pero con disfunción sistólica biventricular leve y diastólica del ventrículo izquierdo con edema pulmonar. Se indicó el tratamiento diurético, con normalización de la función ventricular.

Palabras clave: foramen oval restrictivo, hipertensión pulmonar, recién nacido.

\begin{abstract}
Premature closure of the foramen ovale (FO) or intrauterine restrictive $\mathrm{FO}$ is a rare entity of unknown etiology. This pathology is associated with an elevated risk of pulmonary hypertension, which can cause congestive heart failure with hydrops and fetal death. Patients with restrictive intrauterine FO may develop left ventricle diastolic dysfunction and pulmonary edema.

A newborn with increased right cavities in the prenatal ultrasound is presented. In postnatal echocardiogram, dilatation of right cavities was confirmed with pulmonary hypertension in the systemic range and immobile interatrial septum with closed FO. After 9 days of persistent respiratory distress, an echocardiogram was performed with improvement of pulmonary hypertension but with mild biventricular and diastolic and systolic dysfunction of the left ventricle with pulmonary edema. Diuretic treatment was decided, with normalization of ventricular function.
\end{abstract}

Key words: restrictive foramen ovale, pulmonary hypertension, newborn.

a. Complejo Asistencial Universitario de León, León, España.

Correspondencia:

Lic.Sandra Terroba Seara: sandra_viveiro@hotmail.com

Financiamiento: Ninguno.

Conflicto de intereses: Ninguno que declarar.

Recibido: 30-6-2018

Aceptado: 30-5-2019 http: / / dx.doi.org/10.5546/ aap.2019.e626

Cómo citar: Terroba Seara S, Oulego Erroz I, Lobete Prieto C, Alonso Quintela P. Foramen oval restrictivo intraútero: causa de hipertensión pulmonar neonatal. Arch Argent Pediatr 2019;117(6):e626-e630.

\section{INTRODUCCIÓN}

Durante la vida fetal, el foramen oval (FO) permite que la sangre oxigenada proveniente de la placenta se derive a la aurícula izquierda. ${ }^{1}$ El cierre normal se produce de manera fundamentalmente pasiva, por el incremento del retorno venoso pulmonar y la reducción del sistémico, al disminuir el flujo de la vena cava inferior tras la ligadura del cordón umbilical. ${ }^{2}$

$\mathrm{El}$ cierre prematuro del $\mathrm{FO}$ o $\mathrm{FO}$ restrictivo intraútero es una entidad rara de etiología desconocida que se asocia a hipertensión pulmonar (HTP) en el período neonatal. Uno de los primeros casos fue descrito en 1939, y el primer diagnóstico prenatal de este trastorno fue publicado en 1982 por Hansmann y Redel. ${ }^{3}$

El diagnóstico prenatal debe sospecharse ante la presencia de discrepancia de las cavidades con predominio derecho sin evidencia de cardiopatía estructural. Otros datos que apoyan el diagnóstico son el aneurisma septal atrial, septum primum redundante o hipermóvil, regurgitación tricúspide o ductus arterioso con angulación posterior. ${ }^{3,4}$

Actualmente, existen equipos de ecocardiografía fetal con una alta resolución que permiten visualizar el FO. ${ }^{5}$ Los criterios ecocardiográficos propuestos para el diagnóstico de FO restrictivo incluyen un diámetro del FO inferior a $2 \mathrm{~mm}$ con una velocidad en el doppler superior a $120 \mathrm{~cm} / \mathrm{s}$ y un FO menor de $3 \mathrm{~mm}$ con un gradiente de velocidad doppler medido superior a $5 \mathrm{mmHg}{ }^{3}$

En la mayoría de las ocasiones, el diagnóstico es secundario a un estudio ecocardiográfico realizado por la sospecha de cardiopatía prenatal, como el caso que se presenta a continuación. 
Cuando no existe sospecha prenatal, el diagnóstico suele ser casual en el contexto del estudio de un soplo o de hipoxemia neonatal.

\section{CASO CLÍNICO}

Recién nacido, hijo de madre primípara y sana, de 41 semanas de gestación con peso adecuado para la edad gestacional (peso recién nacido: 3050 g). Diagnosticado en la ecografía prenatal del tercer trimestre con un aumento del tamaño de las cavidades derechas (anillo tricúspide: 13,3 mm; anillo mitral: 10,3 mm; anillo aórtico: 4,6 mm; anillo pulmonar: $6,3 \mathrm{~mm}$ ) sin poder visualizar flujo a través del FO. Se descartó una cardiopatía congénita, incluida la hipoplasia del ventrículo izquierdo. Nació tras un parto eutócico con puntaje de Apgar 8/9 y con un $\mathrm{pH}$ de cordón de 7,14. A los 5 minutos de vida, inició dificultad respiratoria, que requirió oxigenoterapia.

En el ecocardiograma posnatal, se confirmó la dilatación de las cavidades derechas con HTP con presión pulmonar en rango sistémico y se visualizó un septo interauricular inmóvil con FO cerrado sin shunt a través (Figura 1, panel $A$ ).

Asoció una dilatación del seno coronario por la vena cava superior izquierda, ductus arterioso bidireccional con angulación posterior y una comunicación interventricular muscular. Presentó un ventrículo izquierdo y arco aórtico ligeramente hipoplásicos sin datos de coartación (anillo aórtico: $5 \mathrm{~mm}$; anillo mitral: $7 \mathrm{~mm}$; septo interventricular en diástole: 3,9 mm; diámetro diastólico del ventrículo izquierdo: $16 \mathrm{~mm}$; pared posterior del ventrículo izquierdo: $3,6 \mathrm{~mm}$ con fracción de eyección del $76 \%$ ).

Permaneció estable, toleró el cierre ductal sin deterioro hemodinámico, pero persistió la HTP. Durante los primeros 9 días, persistió la taquipnea; precisó la presión positiva continua en las vías respiratorias (continuous positive airway pressure; $C P A P$, por sus siglas en inglés) y oxigenoterapia con fracción inspirada de oxígeno $\left(\mathrm{FiO}_{2}\right)$ de $0,25-0,30$. Se realizó un nuevo ecocardiograma, que evidenció una mejoría de la HTP y se destacó la presencia de disfunción diastólica del ventrículo izquierdo con elevación de la presión de llenado (Figura 1, panel B).

En la ecografía torácica, se observó un patrón de predominio de línea B compatible con edema pulmonar (Figura 2), por lo que se decidió añadir
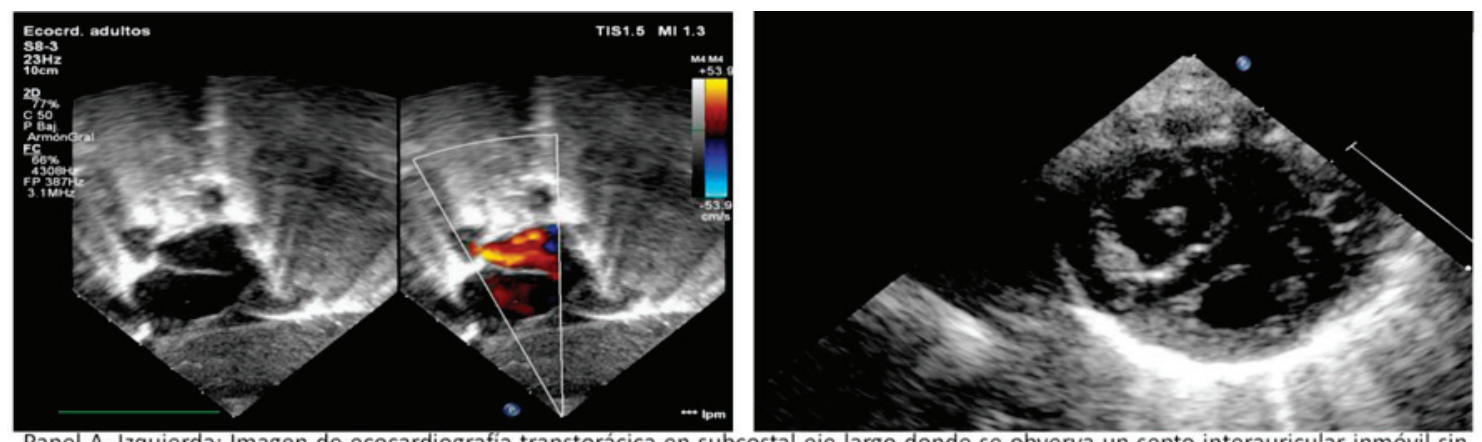

Panel A. Izquierda: Imagen de ecocardiografía transtorácica en subcostal eje largo donde se obverva un septo interauricular inmóvil sin foramen oval ni cortocircuito en el Doppler color. B: Imagen de ecocardiografía transtorácica en paraesternal eje corto donde se observa dilatación de ventrículo derecho con datos de aumento de la presión pulmonar (septo interventricular rectificado en sistole).
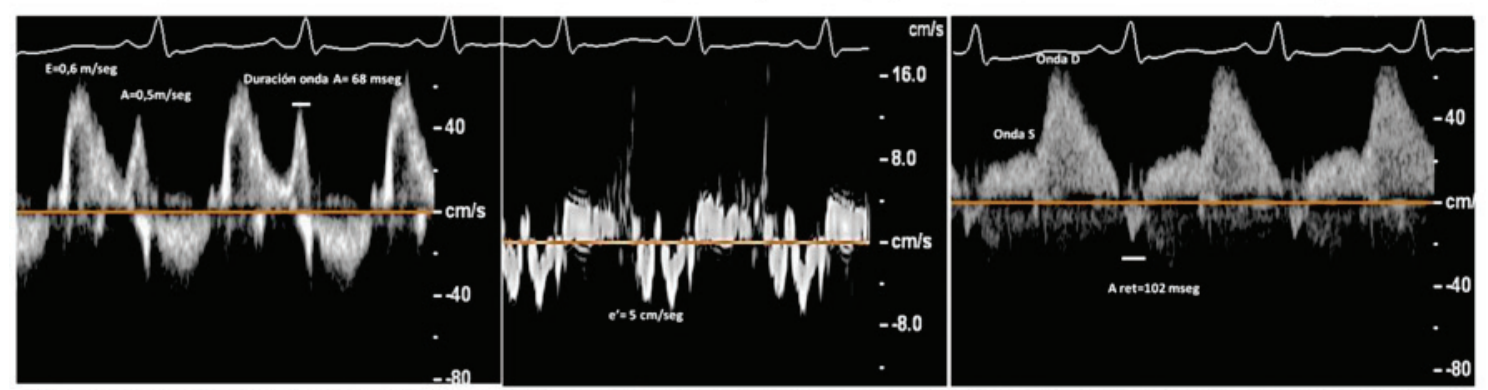

Panel B. Ecografía a los 9 días de vida con datos de disfunción diastólica y elevación de la presión de llenado. Izquierda: Patrón de llenado mitral con alteración de relajación (Onda E > A) y prolongación del tiempo de deceleración de la onda A mitral. Centro: Doppler tisular mitral lateral con alteración de la relajación ventricular con onda e'<á y cociente E/é $=12$. Derecha: Doppler de vena pulmonar con onda $\mathrm{S}<\mathrm{D}$ y duración de la onda a retrógrada mayor que la onda A mitral. 
un tratamiento diurético con furosemida y espironolactona. Tras 4 días de tratamiento, pudo retirarse la oxigenoterapia y persistió la taquipnea, junto con la mejoría de los datos ecográficos con resolución de la HTP.

A los 17 días de vida, fue dado de alta con tratamiento diurético en descenso, sin necesidad de oxigenoterapia y con taquipnea durante la alimentación. En el control en la consulta de Cardiología Infantil a los dos meses, se comprobó la normalización de la función ventricular y se retiró el tratamiento diurético sin presentar empeoramiento en los controles posteriores.

\section{COMENTARIO}

La restricción del flujo en el FO durante la vida fetal tiene efectos hemodinámicos diversos en función de la gravedad y el momento de la gestación en que acontezca. La consecuencia directa del cierre del FO es el aumento de flujo sanguíneo pulmonar y del gasto cardíaco derecho respecto al sistémico, favorecido, en gran parte, por el aumento de la oxigenación en la arteria pulmonar, lo que produce una dilatación progresiva de las cavidades derechas. ${ }^{1}$

Cuando el cierre del FO es precoz, la restricción al flujo a través del foramen puede ser tan grave que determina el desarrollo de un síndrome de corazón izquierdo hipoplásico. ${ }^{4,5} \mathrm{Sin}$ embargo, cuando la restricción es más tardía, el cuadro está dominado por la dilatación derecha y puede presentarse hidrops fetal en algunos casos graves.

\section{FigURA 2}

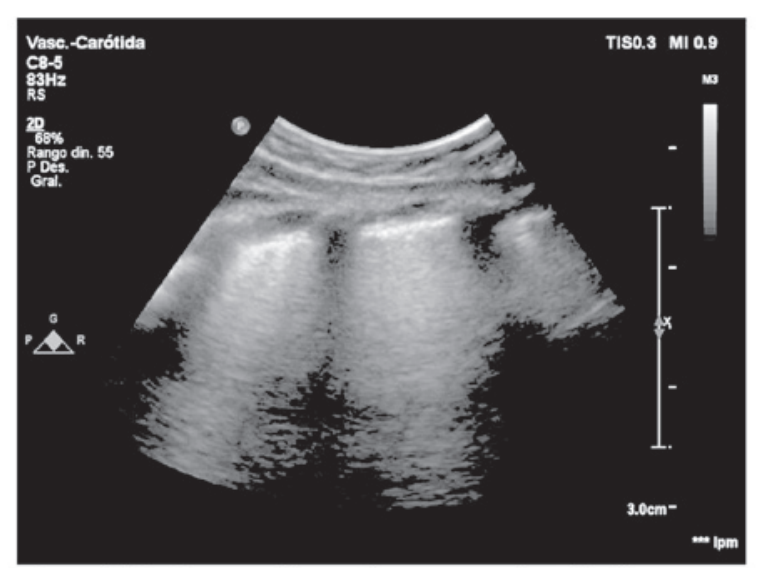

Panel C. Ecografía pulmonar a los 9 días con un patrón de líneas B coalescente compatible con edema intersticial, en relación con la disfunción diastólica.
Cuando la gestación llega a término, la clínica neonatal está dominada por la HTP. A pesar de que no existe una evidencia clara de que el cierre prematuro de FO sea una causa de enfermedad pulmonar vascular obstructiva, existe algún estudio que sugiere esta posibilidad. ${ }^{6}$

El aumento del flujo en las arterias pulmonares podría originar el remodelado y aumento de las resistencias pulmonares y ser la causa del cuadro de persistencia de circulación fetal neonatal. Normalmente, el curso de la HTP es transitorio y más leve que el de otras causas de HTP neonatal.

Otros hallazgos asociados al FO restrictivo son taquicardia supraventricular, regurgitación tricuspídea, derrame pericárdico, falla cardíaca congestiva, incluso muerte fetal y/o neonatal. Cabe destacar la alta incidencia de anomalías extracardíacas en la literatura (Tabla 1).

Como novedad, en nuestro caso, además de la HTP descrita asociada al FO restrictivo, se observó una disfunción diastólica del ventrículo izquierdo con edema pulmonar, que precisó tratamiento diurético y soporte respiratorio. Es probable que esta situación traduzca una disminución de la distensibilidad del ventrículo izquierdo fruto de una restricción del flujo al ventrículo en una etapa tardía de la gestación.

Como comentarios finales, cabe decir que el diagnóstico prenatal de FO restrictivo debe sospecharse ante la presencia de discrepancia de cavidades con sobrecarga derecha sin cardiopatía asociada y debe intentar confirmarse mediante la visualización del FO.

El FO restrictivo debe considerarse en el diagnóstico diferencial de la HTP neonatal "idiopática". Se debe tener en cuenta la función del ventrículo izquierdo en estos casos, ya que puede existir disfunción diastólica significativa, lo cual tiene implicaciones en el tratamiento de la HTP.

\section{REFERENCIAS}

1. Uzun O, Babaoglu K, Ayhan Y, Moselhi M, et al. Diagnostic ultrasound features and outcome of restrictive foramen ovale in fetuses with structurally normal hearts. Pediatr Cardiol. 2014; 35(6):943-52.

2. Maroto Monedero C, Camino López M, Girona JM, Malo Concepción P. Guías de práctica clínica de la Sociedad Española de Cardiología en las cardiopatías congénitas del recién nacido. Rev Esp Cardiol. 2001; 54(1):49-66.

3. Gupta U, Abdulla RI, Bokowski J. Benign outcome of pulmonary hypertension in neonates with a restrictive patent foramen ovale versus result for neonates with an unrestrictive patent foramen ovale. Pediatr Cardiol. 2011; 32(7):972-6. 
TABLA 1. Bibliografía reciente sobre foramen oval restrictivo

\begin{tabular}{|c|c|c|c|c|c|}
\hline Publicación & Año & Casos & Hallazgo prenatal & Hallazgo posnatal & Evolución \\
\hline Umang Gupta et al. & 2011 & 7 & & HTP & Tratamiento \\
\hline Iwamoto et al. & 2011 & 1 & $\begin{array}{l}\text { Válvula mitral pequeña } \\
\text { + hipoplasia de la aorta } \\
\text { ascendente } \\
\text { + FO cerrado ( } 37 \text { sem.) }\end{array}$ & $\begin{array}{l}\text { HTP -> vasodilatador } \\
\text { y oxigenoterapia }\end{array}$ & $\begin{array}{c}14 \text { meses, reemplazo } \\
\text { mitral -> HTP } \\
\text { posquirúrgica, } \\
\text { tratamiento con } \\
\text { bosentan, sildenafilo } \\
\text { y oxigenoterapia }\end{array}$ \\
\hline \multirow[t]{2}{*}{ Yi-Dan Li et al. } & 2013 & 1 & $\begin{array}{l}\text { Flujo estrecho I-D } \\
\text { en atrium ( } 37 \text { sem.) }\end{array}$ & Apgar bajo & Fallecido a las 24 horas \\
\hline & & 1 & $\begin{array}{c}\text { FO restrictivo }+ \\
\text { CIV membranosa }+ \\
\text { hipoplasia de la aorta ascendente }\end{array}$ & $\begin{array}{l}\text { FO cerrado sin } \\
\text { otras anomalías }\end{array}$ & Asintomático \\
\hline \multirow[t]{13}{*}{ Orhan Uzun et al. } & 2014 & 1 & $\begin{array}{l}\text { Dilatación del VD + } \\
\text { SP redundante e hipermóvil } \\
\text { + DA posterior }\end{array}$ & НТР & Oxigenoterapia \\
\hline & & 1 & $\begin{array}{c}\text { Dilatación del VD + } \\
\text { SP aplanado + arritmias + } \\
\text { DA posterior }\end{array}$ & $\begin{array}{l}\text { Hipertrofia del VD + } \\
\text { disfunción VI }\end{array}$ & Asintomático \\
\hline & & 1 & $\begin{array}{c}\text { Dilatación del VD + } \\
\text { arritmias + SP aplanado + } \\
\text { DA posterior }\end{array}$ & $\begin{array}{c}\text { HTP + disfunción del VI + } \\
\text { + disfunción del VD + } \\
\text { displasia de la válvula } \\
\text { tricuspídea }\end{array}$ & $\begin{array}{c}\text { Síndrome de Alstrom }+ \\
\text { WPW -> } \\
\text { tratamiento } \\
\text { intensivo }\end{array}$ \\
\hline & & 1 & $\begin{array}{l}\text { Dilatación del VD + } \\
\text { SP redundante e hipermóvil + } \\
\text { DA posterior }\end{array}$ & HTP & $\begin{array}{l}\text { Hipotiroidsimo + } \\
\text { enf. celíaca -> } \\
\text { oxigenoterapia }\end{array}$ \\
\hline & & 7 & $\begin{array}{c}\text { Dilatación del VD + } \\
\text { SP redundante }+ \\
\text { DA anterior/ posterior }\end{array}$ & Normal & Asintomático \\
\hline & & 4 & $\begin{array}{l}\text { Dilatación del VD + } \\
\text { SP redundante e hipermóvil } \\
\text { + DA posterior }\end{array}$ & Normal & Asintomático \\
\hline & & 2 & $\begin{array}{l}\text { Dilatación del VD + } \\
\text { SP redundante e hipermóvil } \\
\text { + DA anterior }\end{array}$ & Normal & Asintomático \\
\hline & & 1 & $\begin{array}{l}\text { Dilatación del VD + } \\
\text { SP redundante e hipermóvil } \\
\text { + DA posterior }\end{array}$ & Normal & Síndrome de Down \\
\hline & & 1 & $\begin{array}{l}\text { Dilatación del VD + } \\
\text { SP redundante } \\
\text { + DA posterior }\end{array}$ & HTP + disfunción del VI & $\begin{array}{l}\text { Síndrome Vacterl } \\
\text { Fallecido }\end{array}$ \\
\hline & & 1 & $\begin{array}{l}\text { Dilatación del VD + } \\
\text { SP redundante e hipermóvil + } \\
\text { DA anterior }\end{array}$ & НТР & Oxigenoterapia \\
\hline & & 1 & $\begin{array}{l}\text { Dilatación del VD + } \\
\text { SP redundante }+ \\
\text { DA posterior }\end{array}$ & Normal & $\begin{array}{c}\text { Síndrome de Menkes -> } \\
\text { Fallecido }\end{array}$ \\
\hline & & 1 & $\begin{array}{l}\text { Dilatación del VD + } \\
\text { SP redundante e hipermóvil } \\
+ \text { DA anterior }\end{array}$ & DAP grave & Cierre del DAP \\
\hline & & 1 & $\begin{array}{l}\text { Dilatación del VD + } \\
\text { SP redundante e hipermóvil } \\
+ \text { DA anterior }\end{array}$ & $\begin{array}{c}\text { HTP + hipertrofia del VD + } \\
\text { displasia de la válvula } \\
\text { tricuspídea }\end{array}$ & $\begin{array}{l}\text { Tratamiento } \\
\text { intensivo }\end{array}$ \\
\hline
\end{tabular}

HTP: hipertensión pulmonar; FO: foramen oval; CIV: comunicación intraventricular; VD: ventrículo derecho; VI: ventrículo izquierdo; SP: septum primum; DA: ductus arterioso; VACTERL: vértebras, ano, corazón, fístula traqueoesofágica, riñón y extremidades inferiores; WPW: Wolff-Parkinson-White. 
e630 / Arch Argent Pediatr 2019;117(6):e626-e630 / Presentación de casos clínicos

4. Hagen A, Albig M, Schmitz L, Hopp H, et al. Prenatal diagnosis of isolated foramen ovale obstruction. A report of two cases. Fetal Diagn Ther. 2005; 20(1):70-3.

5. $\mathrm{LiYD}, \mathrm{LiZA}, \mathrm{He} Y \mathrm{H}$. Premature closure or restriction of the foramen ovale. prenatal diagnosis by directional enhanced flow imaging. J Ultrasound Med. 2013; 32(7):1291-4.
6. Iwamoto Y, Tamai A, Kawasaki H, Taketazu M, et al. Late clinical manifestations of mitral valve disease and severe pulmonary hypertension in a patient diagnosed with premature closure of foramen ovale during fetal life. World J Pediatr. 2011; 7(2):182-4 